\title{
Spontaneous inferior lumbar hernia: rare case report and review
}

\begin{abstract}
Lumbar hernias are uncommon and few cases have been reported till date. They commonly occur due to trauma, surgery and infection. Incidence has increased after motor vehicle collision injuries. However, spontaneous lumbar hernias that too in inferior triangle is rare. It is treated with different surgical approaches and techniques. We report a case of primary spontaneous inferior lumbar hernia which was repaired by open surgical approach using Vypro (polypropylene). Recent studies have demonstrated the advantages of a laparoscopic repair instead of the classic open approach as the ideal treatment option for lumbar hernias.
\end{abstract}

Keywords: lumbar hernias, trauma, laparoscopic repair, abdominal contents, semispherical bulge
Volume 6 Issue 2 - 2018

\section{Sunder Goyal,' Sanjeev Singla, ${ }^{2}$ Dewat Ram Nakipuria ${ }^{3}$ \\ 'Department of Surgery, ESIC Medical College, India \\ ${ }^{2}$ Department Surgery, BPS Govt med college for Women, India \\ ${ }^{3}$ Department Surgery, North DMC Medical College, India}

Correspondence: Sunder Goyal, Department of Surgery, ESIC Medical College, India, Email goyal.sunder@yahoo.in

Received: January 29, 2018 | Published: April 09, 2018

\section{Introduction}

Lumbar hernias area protrusion of intra abdominal contents through a weakness or rupture in the posterior abdominal wall. Lumbar hernias are rare among all other hernias. ${ }^{1}$ Due to advance diagnostic tools, lumbar hernias are diagnosed more frequently in current practice since then, only about 300 cases have been reported. ${ }^{2}$ Petit described the inferior lumbar triangle in 1783 and Grynfeltt described the superior lumbar triangle in 1866; both are anatomical boundaries where $95 \%$ of lumbar hernias occur, whereas the other $5 \%$ are considered to be diffuse. Twenty percent of lumbar hernias are congenital and the other $80 \%$ are acquired; the acquired lumbar hernias can be further classified into either primary (spontaneous) or secondary. ${ }^{3,4}$ Primary spontaneous lumbar hernias are the rarest among all the presentations. Usually lumbar hernias present as protruding semispherical bulge in back of lumbar region. Rarely, can

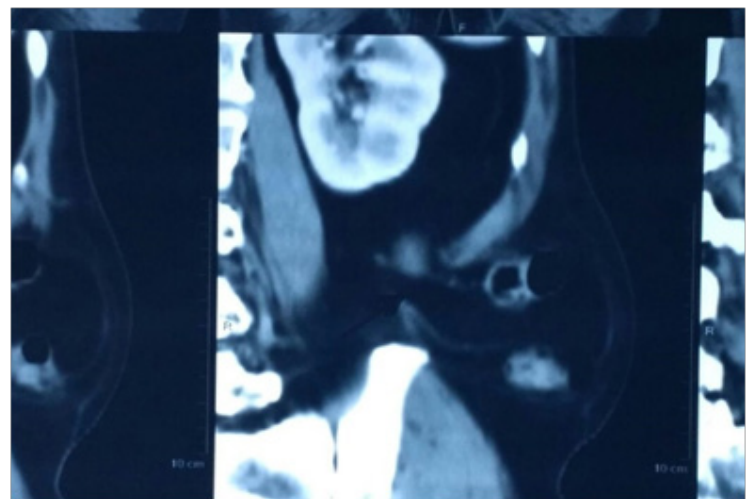

Figure I CT scan showing hernia.

\section{Discussion}

Both lumbar triangles are bound superiorly by the $12^{\text {th }}$ rib, inferiorly by the iliac crest, medially by the erector spinae muscle group, and laterally by the posterior border of the external oblique muscle., ${ }^{3,4}$ Weakness and defects can occur in the musculature/aponeurosis of lumbar region. Defect may be congenital, spontaneous, or traumatic. present with an incarcerated or strangulated bowel. Computerized tomography (CT) is the "gold standard" for diagnosing a lumbar hernia as it is able to define muscular and fascial layers and thus can detect a defect in one or more of these layers. It can also evaluate the presence of herniated contents.

\section{Case report}

A 50 year-old male presented with progressively enlarging swelling in the right loin. There was no history of trauma, fever, weight loss and any associated bowel or urinary complaint. There was no significant past medical or surgical history. On examination there was a $5 \mathrm{~cm} \times$ $4 \mathrm{~cm}$ partially reducible non tender mass located in the inferior lumbar triangle which was prominent during Valsalvamaneuver. Computer tomography (CT) scan was done which confirmed the diagnosis of inferior lumbar hernia (Figure 1). Open repair done with meshplasty (Figure 2).

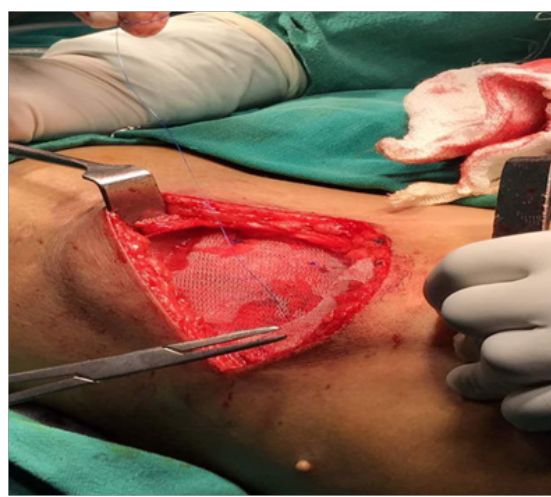

Figure 2 Mesh repair after hernia repair.

Congenital hernias can develop in infants due to arrest or abnormal musculoskeletal development, ${ }^{5}$ Spontaneous hernias occurs mostly in the superior triangle of Grynfeltt-Less haft represent and incidence is about approximately $50 \%$ of all lumbar hernias. ${ }^{6}$ Traumatic lumbar hernias are caused by postoperative flank incisions as well as nonoperative factors including crushing or penetrating injuries, falls, or post-inflammatory states and incidence is about $25 \%$ of all cases. ${ }^{3,4}$ 
Both spontaneous and traumatic types show a predilection for the left side than right side. These are more common in males and are most common in patients between ages 50 and 70 .

Primary lumbar hernias present most commonly as a palpable mass which increases in size on coughing. They are usually reducible and may disappear in supine position. Patients may present with nonspecific abdominal pain or back pain which may represent sciatica. There are occasions when lumbar hernias may lead to bowel obstruction and sometimes urinary obstruction resulting in hydronephrosis. The most common differential diagnoses are lipomas, fibromas, hematomas, abcesses, intra-abdominal/retroperitoneal tumors and panniculitis.

X-rays may not be helpful in diagnosis and CT scan remains the gold standard. CT has been used in the past in the diagnosis of inguinal, obturator, diaphragmatic, and Spigelian hernia. CT is able to define muscular and fascial layers and the presence of herniated fat and/or viscera. Lumbar hernia may contain only extraperitoneal fat, extraperitoneal fat plus kidney or colon, as well as intraperitoneal structures (most commonly small bowel). When muscular layers are intact, CT is the only radiographic method necessary for diagnosis. When hernias do exist, CT can show which fascial or muscular layers are involved and the content of the hernial sac. A normal CT of this region in a symptomatic patient enables the physician to confidently exclude a lumbar hernia and guide therapy away from dealing with a structural abnormality. In selected patients MRI also has a role in diagnosing and planning the surgical treatment. ${ }^{?}$

If defect is narrow then these hernias may present as surgical emergencies (about 9\%). ${ }^{8}$ Surgery is treatment of choice. Earlier, most of the lumbar hernias were repaired primarily using muscle flaps from gluteus major and medius muscle, latissimus dorsi muscle and fascia lata. ${ }^{9}$ Due to poor fascial strength and high tension of the repair, the recurrence rate was very high. These days, artificial mesh with polypropylene, Marlex, or prolene was used to bridge the defect. The Dowd-Ponka technique of repairing lumbar hernias involves making an incision over the hernia site and reducing the contents to the abdomen, followed by covering the defect with prosthetic mesh which is sutured to the external oblique, latissimus dorsi and the lumbar periostium. This is followed by constructing a gluteal fascial flap to cover the defect approximating the external oblique and latissimus dorsi muscles. ${ }^{10}$

In 1996 the first trans-abdominal laparoscopic approach was introduced by Burick and Parascandola. ${ }^{11}$ The balloon dissector was used in 1999 for total extra-peritonial approach which was described by Woodward et al. ${ }^{12} \mathrm{~A}$ paper published in 2005 shows a prospective study of lumbar hernia repairs classical versus laparoscopic approaches. It has shown statistically significant lower morbidity rates, shorter length of hospital stay, reduced analgesics requirement and earlier return of normal activity in the laparoscopic group. ${ }^{13}$ Synthetic mesh for hernia repair has the complications of Chances of infection, bowel obstruction and fistula formation are high with synthetic mesh, so, biosynthetic mesh made of human acellular dermis has replaced the synthetic mesh with good results especially in contaminated wounds. ${ }^{14}$

\section{Conclusion}

Lumbar hernias are rare. CT scan is gold standard in diagnosis. Open as well as laparoscopic approaches are used for treatment. Laparoscopic approach is treatment of choice. Open surgery is done in patients with large defects or after failed laparoscopic approach.

\section{Acknowledgements}

None.

\section{Conflict of interest}

The authors declared that there are no conflicts of interest.

\section{References}

1. Hafner C, Wylie J, Brush BE. Petit's lumbar hernia: repair with Marlex mesh. Arch Surg. 1963;86:180-186.

2. Moreno Egea A, Baena EG, Calle MC, et al. Controversies in the current management of lumbar hernias. Arch Surg. 2007;142(1):82-88.

3. Garangeot RJ. Colon traited'operation. Chirurgie. 1731;1:369-370.

4. Stamatiou D, Skandalakis JE, Skandalakis LJ, et al. Lumbar hernia: surgical anatomy, embryology, and technique of repair. Am Surg. 2009;75(3):202-207.

5. Heniford BT, Iannitti DA, Gagner M. Laparoscopic inferior and superior lumbar hernia repair. Arch Surg. 1997;132(10):1141-1144.

6. Grynfeltt J. Quelques mots sur la hernielombaire. Montp Med. $1866 ; 16: 323$

7. Thilan B Walgamage, Ramesh BS, Yaqoob Alsawafi. Case report and review of lumbar hernia. Int J Surg Case Rep. 2015;6:230-232.

8. Alfredo ME, Enrique GB, Miquel CC, et al. Controversies in the current management of lumbar hernias. Arch Surg. 2007;142(1):82-88.

9. Swartz DWT. Lumbar hernia. In: Nyhus LM, Condon RE, editors. Hernia. $2^{\text {nd }}$ ed. Lippincott: Philadelphia; 1978. p. 409-426.

10. Skandalakis LJ, Skandalakis JE, Skandalakis PN. Surgical anatomy and technique 3rd ed. Springer; 2008.

11. Burick AJ, Parascandola S. Laparoscopic repair of a traumatic lumbar hernia: a case report. J Laparoendosc Surg. 1996;6(4):259-262.

12. Woodward AM, Flint L, Ferrara J. Laparoscopic retroperitoneal repair of recurrent postoperative lumbar hernia. J LaparoendoscAdv Surg Tech A. 1999;9(2):181-186.

13. MorenoEgea A, Torralba JA, Morales G. Open vs laparoscopic repair of secondary lumbar hernias: a prospective nonrandomized study. Surg Endosc. 2005;19(2):184-187.

14. Beth Ann S, Victoriya Sara C, Kelly J, et al. Repair of a traumatic lumbar hernia with biosynthetic mesh: a novel approach and review of the literature. J Curr Surg. 2012;2(3):105-109. 